\title{
The Impact of the Earth's Movement through the Space on Measuring the Velocity of Light (Part Two)
}

\author{
Miloš Čojanović \\ Independent Researcher, Montreal, Canada \\ Email: cojmilmo@gmail.com
}

How to cite this paper: Čojanović, M. (2017) The Impact of the Earth's Movement through the Space on Measuring the Velocity of Light (Part Two). Journal of Applied Mathematics and Physics, 5, 741-757.

https://doi.org/10.4236/jamp.2017.53063

Received: February 20, 2017

Accepted: March 28, 2017

Published: March 31, 2017

Copyright $\odot 2017$ by author and Scientific Research Publishing Inc.

This work is licensed under the Creative

Commons Attribution International

License (CC BY 4.0).

http://creativecommons.org/licenses/by/4.0/

\begin{abstract}
In this paper is presented an experiment that has a goal to measure the speed of light in one direction, using one clock and one mirror. To achieve this, we have to make the measurements during the period of one year (nine months at least), taking into account earth's movement through the space, earth's rotation around its axis and the fact that earth spin axis is tilted with respect to the plane of its orbit of the sun.
\end{abstract}

\section{Keywords}

Speed of Light, One Way Speed of Light, Absolute Velocity of the Earth

\section{Introduction}

The paper [1] described a method to measure the speed of light in the direction of the western-east using one clock and one mirror,without the assumption that speeds of light in two opposite direction are equal.

We have to give some explications regarding the paper [1]. "The projection of the vector $\mathbf{v}$ on $\mathbf{z}$ axis is denoted by $\mathbf{v}_{z}$ (actually $\boldsymbol{v}_{2}+\boldsymbol{v}_{3}$, because $\boldsymbol{v}_{1}$ is perpendicular on $\mathrm{z}$ axis)."

Generally, vector $\boldsymbol{v}_{1}$ (earth's motion around the sun) is not perpendicular on the $\boldsymbol{z}$ axis, thus this condition is absolutely unnecessary. Actually, the experiments that are explained in [1] could be performed at any time without waiting for the certain period in the year when vector $v_{1}$ is perpendicular to the $z$ axis. Later, in this paper we will talk more about this subject.

The one way of speed of light will be measured in three different directions. On the basis of these results, under certain conditions, the speed of light will be derived in any direction. 
First,let us to mention and clarify some definitions that were already given in the paper [1].

To the earth's movement through the space we will join a vector $\boldsymbol{V}$

$$
\boldsymbol{v}=v_{1}+v_{2}+v_{3}
$$

$\boldsymbol{v}_{1}$-represents earth's motion around the Sun

$v_{2}$-represents sun's (solar system) motion around the centre of the Milky Way

$\boldsymbol{v}_{3}$-represents Milky Way's motion about (from, to) some point (or other galaxies) in the universe.

Now, we are going to define a coordinate system. The center of the earth $\boldsymbol{O}$ is going to be an origin of the coordinate system. Earth's axis of rotation in the direction South-North is going to be positive z-axis.

Positive $\mathrm{z}$ axis is denoted by $\mathbf{z}^{+}$and negative $\mathrm{z}$ axis by $\mathbf{z}^{-}$. Same convention will be applied to the $\mathrm{x}$ and $\mathrm{y}$ axes.

Earth's equatorial plain, denoted by $E$ is going to be $x y$ plane. Let $R$ represent the earth' orbital plane around the Sun and let $n$ represent a line through the point $O$ orthogonal to $R$. Let $\varphi_{0}$ denotes angle between $n$ and $z$ (earth's axial tilt).

$$
\varphi_{0}=\frac{23.5^{\circ} \pi}{180^{\circ}}
$$

Lines $z$ and $n$ determine a plane $N$ that will be $x z$ plane Intersection between planes $E$ and $N$ determine a line, that is going to be $\mathrm{x}$ axis.

Let $p$ denote an intersection line between the planes $R$ and $N$. Normal projection of the $x$ on the plane $R$, and normal projection of the $\mathrm{z}$ on the plane $R$ conicide to $p$. Let $p^{+}$denote normal projection of the $\mathbf{z}^{+}$, and $p^{-}$denote normal projection of the $\mathbf{z}^{-}$on the plane $R$.

Positive $\boldsymbol{x}^{-}$axis denoted by $\boldsymbol{x}^{+}$is going to be chosen thus its normal projection on the plane rcoincides to $p^{+}$. It follows that an angle between $\boldsymbol{x}^{+}$ and plane $R$ is equal to $\varphi_{0}$.

$$
\angle\left(x^{+}, R\right)=\angle\left(x^{+}, p^{+}\right)=\varphi_{0}
$$

Let $\varphi_{1}$ denotes angle between $\mathbf{z}^{+}$and plane $R$.

$$
\varphi_{1}=\angle\left(\mathbf{z}^{+}, R\right)=\angle\left(\mathbf{z}^{+}, p^{+}\right)=\frac{\Pi}{2}-\varphi_{0}
$$

Planes $E$ and $R$ are two distinct planes with common point $O$ thus their intersection is a straight line that is denoted by $y^{\prime}$. Our $\boldsymbol{x} \boldsymbol{z}$ plane is orthogonal to the both planes $E$ and $R$, so it's perpendicular to their intersection $y^{\prime}$. In that way it's natural to take $y^{\prime}$ as $\boldsymbol{y}$-axis.

It is worth mentioning that $y$-axis lies in the plane $R$.

Positive axis $y$ will be chosen in the way that $O x y z$ forms right-handed coordinate system.

The center $O$ moves along the earth's orbit $(E)$ but $x, y, z$ axes remain unchanged (if we consider them as vectors), because during a period of one year 
we will assume that positive $Z$-axis and orthogonal line $n$ on the plane $R$ are invariant under the earth's movement.

In that way the earth-centered inertial axis frame has been defined [2].

During the period of one year we will assume that vectors $\boldsymbol{v}_{2}$ and $\boldsymbol{v}_{3}$ remain unchanged. Vector $\boldsymbol{v}$ can be split into the two parts. The first one $v_{1}$ that changes during the experiment and the constant part equal to $\left(\boldsymbol{v}_{2}+\boldsymbol{v}_{3}\right)$.

Vector $v_{1}$ can be expressed as a function of time

$$
\boldsymbol{v}_{1}=\boldsymbol{v}_{1}(T) \text { where }(0 \leq T \leq 365.24219 \text { days })
$$

Let $\boldsymbol{u}$ denote the the sum of the vectors $\boldsymbol{v}_{2}$ and $\boldsymbol{v}_{3}$

$$
\boldsymbol{u}=\boldsymbol{v}_{1}+\boldsymbol{v}_{2}
$$

The orthogonal projections of the vector $\boldsymbol{u}$ on the $\boldsymbol{x}, \boldsymbol{y}, \mathbf{z}$-axes are denoted by $u_{x}, u_{y}, u_{z}$ respectively.

$$
\begin{aligned}
& u_{x}=\operatorname{proj}_{x}(\mathbf{u}) \\
& u_{y}=\operatorname{proj}_{y}(\mathbf{u}) \\
& u_{z}=\operatorname{proj}_{z}(\mathbf{u})
\end{aligned}
$$

We will also suppose that the speed of light in the direction $A B$ which is denoted by

$$
\mathbf{c}_{A B}=\mathbf{c}-|\mathbf{v}| * \cos (\varphi)
$$

where

$A B$-Is some arbitrary line in the direction west-east or south-north.

$c$-Represents velocity of light in vacuum for a body at rest.

$\boldsymbol{v}$-Velocity of the earth through the space with the respect to the some point in the universe. (This is our hypothesis.)

$\varphi$-Angle between $v$ and $\mathrm{AB}$.

Depending on the outcome of the experiment we will distinguish three cases:

i) We will say that the experiment is "negative" if the speed of the light does not depend on the direction in which it has been measured $\left(\boldsymbol{c}_{A B}=\boldsymbol{c}_{B A}\right)$. In this case, the speed of light is invariant to the earth movement through the space,

ii) We will say that the experiment is "positive" if the speed of the light depends on the direction in which it has been measured $\left(\boldsymbol{c}_{\mathrm{AB}} \neq \boldsymbol{c}_{\mathrm{BA}}\right)$. In this case, it is possible to derive direction and magnitude of the earth movement with the respect to the coordinate system $O x y z$.

iii) We will say that experiment failed if we are not able to say that outcome of the experiment is either "positive" or "negative".

\section{Planning an Experiment}

First, we are going to define some important points that lay on the earth's orbit about the sun.

The $\boldsymbol{y}$-axis lies in the plane $R$, thus its orthogonal projection $y^{\prime}$ on the plane $R$ coincides to $\mathrm{y}$, Orthogonal projections of the $\boldsymbol{x}$ and $\mathbf{z}$-axis on the plane $R$ lay on the line $p$. During the period of one year vector $v_{1}(T)$ will ro- 
tate about the origin $\mathrm{Oby} 2 * \Pi$.

Thus, for any non-zero vector in the plane $R$ there is a point on the earth's orbit around the sun at which that vector and $v_{1}(T)$ have the same direction.

We will say that two vectors have the same direction if an angle between them is equal to zero.

Projection of the vector $\boldsymbol{v}_{1}(T)$ on the $\boldsymbol{x}^{+}$-axis is given by the expression

$$
\begin{aligned}
\operatorname{proj}_{X}\left(\boldsymbol{v}_{1}(T)\right) & =\left|\boldsymbol{v}_{1}(T)\right| * \cos \left(\angle\left(\boldsymbol{p}^{+}, \boldsymbol{v}_{1}(T)\right)\right) * \cos \left(\angle\left(\boldsymbol{p}^{+}, \boldsymbol{x}^{+}\right)\right) \\
& =\left|\boldsymbol{v}_{1}(T)\right| * \cos \left(\angle\left(\boldsymbol{p}^{+}, \boldsymbol{v}_{1}(T)\right)\right) * \cos \left(\varphi_{0}\right)
\end{aligned}
$$

Obviously $\operatorname{proj}_{x}\left(\boldsymbol{v}_{1}(T)\right)$ has a maximum magnitude when $\boldsymbol{p}^{+}$and $\boldsymbol{v}_{1}(T)$ have the same direction.

A similar reasoning can be applied to the other axes.

Let us denote by $X_{+}$a point on the earth's orbit around the Sun at which $\boldsymbol{v}_{1}(T)$ and orthogonal projection of $\boldsymbol{x}^{+}$on the plane $R$ have the same direction;

Let us denote by $Z_{+}$a point on the earth's orbit around the Sun at which $\boldsymbol{v}_{1}(T)$ and orthogonal projection of $\mathbf{z}^{+}$on the plane $\mathrm{R}$ have the same direction;

It follows that the point $Z_{+}$coincides to the point $X_{+}$.

A moment when the earth reaches to the point $X_{+}\left(Z_{+}\right)$will be denoted by $T_{x+}\left(T_{z+}\right)$.

Let denote by $Y_{+}$a point on the earth's orbit around the Sun at which $v_{1}(T)$ and $y^{+}$have the same direction.

A moment when the earth reaches to the point $Y_{+}$will be denoted by $T_{y+}$.

Let us denote by $X_{-}$a point on the earth's orbit around the Sun at which $\boldsymbol{v}_{1}(T)$ and orthogonal projection of $\boldsymbol{x}^{-}$on the plane $R$ have the same direction.

Let us denote by $Z_{-}$a point on the earth's orbit around the Sun at which $\boldsymbol{v}_{1}(T)$ and orthogonal projection of $\mathbf{z}^{-}$on the plane $R$ have the same direction.

It follows that the point $Z_{-}$coincides to the point $X_{-}$.

A moment when the earth reaches to the point $X_{-}\left(Z_{-}\right)$will be denoted by $T_{x-}\left(T_{z^{-}}\right)$.

Let us denote by $Y_{-}$a point on the earth's orbit around the Sun at which $\boldsymbol{v}_{1}(T)$ and $\boldsymbol{y}^{-}$have the same direction.

A moment when the earth reaches to the point $Y_{-}$will be denoted by $T_{y_{-}}$.

Now, we will establish relation between the distance between the points $A$ and $B$ denoted by $L$ and the accuracy of the clock that is used in the experiment.

Time $t_{1}$ that it takes for a signal to travel from $A$ to $B$ is qiven by expression

$$
t_{1}=\frac{L}{(c-v)} \quad(\text { from 1.10) }
$$

Time $t_{2}$ that it takes for a signal to travel from $B$ to $A$ is given by

$$
t_{2}=\frac{L}{c+v} \quad(\text { from } 1.10)
$$


Therefore it follows that the difference between $t_{2}$ and $t_{1}$ is given by

$$
\left|t_{1}-t_{2}\right|=\left|\frac{L}{(c-v)}-\frac{L}{c+v}\right|=\frac{2 L v}{c^{2}-v^{2}} \sim \frac{2 L v}{c^{2}} \quad\left(c^{2} \gg v^{2}\right)
$$

Obviously the clock accuracy must be high enough in order the difference between times $t_{1}$ and $t_{2}$ could be detected.

Let the clock accuracy is equal to $a^{*} 10^{-p} \sec (1<a<10, p \in N)$.

It follows that

$$
\left(\frac{2 L v}{c^{2}}\right) \gg a^{*} 10^{-p} \Rightarrow\left|c \approx 300000 \frac{\mathrm{km}}{\mathrm{sec}}, v>30 \frac{\mathrm{km}}{\mathrm{sec}},\right| \Rightarrow p \gg 9-\frac{\ln \left(\frac{2 * L}{3^{*} a}\right)}{\ln (10)}
$$

(we assume that $v=30 \mathrm{~km} / \mathrm{sec}$ represents some minimum value for $v$ )

( $L$ is given in $\mathrm{km}$ )

The principle (5) must be respected in this experiment.

\section{The Measurements along Z-Axis}

First we are going to explain how to make measurements along $z$-axis, because they are simpler then those along $x$ or $y$ axis.

Suppose that points $A$ and are $D$ given, thus point $A$ is the point on the earth's equator and $A D$ lies in the South-North direction. In other words $A D$ is parallel to the $z$-axis, The distance between the points $A$ and $D$ is denoted by $L$.

From the point $A$ we are going to send a signal towards point $D$. After the signal reaches point $D$ it will be reflected back to the point $A$.

Let $t$ represent the time that it takes for the signal to travel from the point $A$ to the point $D$ and back to the point $A$.

The first measurement is going to be performed when earth reaches to the point $Z_{+}\left(X_{+}\right)$That moment is already denoted by $T_{z_{+}}\left(T_{x_{+}}\right)$.

Let $u_{0}$ represent the orthogonal projection of vector $v_{1}\left(T_{z+}\right)$ on the $z$ axis.

$$
\begin{aligned}
u_{0} & =\operatorname{proj}_{z}\left(\boldsymbol{v}_{1}\left(T_{z+}\right)\right)=\left|\boldsymbol{v}_{1}\left(T_{z+}\right)\right| * \cos \left(\angle\left(\mathbf{z}^{+}, \boldsymbol{v}_{1}\left(T_{z+}\right)\right)\right)=\left|\boldsymbol{v}_{1}\left(T_{z+}\right)\right| * \cos \left(\varphi_{1}\right) \\
& =\left|\boldsymbol{v}_{1}\left(T_{z+}\right)\right| * \sin \left(\varphi_{0}\right) \approx 11.96247 \mathrm{~km} / \mathrm{sec}
\end{aligned}
$$

Let $t_{0}$ represent the time that it takes for the signal to travel from point $A$ to point D and back to point $A$.

$$
t_{0}=\frac{L}{c-\left(u_{0}+u_{z}\right)}+\frac{L}{c+\left(u_{0}+u_{z}\right)}=\frac{2 * L^{*} c}{c^{2}-\left(u_{0}+u_{z}\right)^{2}}
$$

The $T_{y+}$ denotes moment when earth reaches to the point $Y_{+}$. Let $t_{1}$ represent the time that it takes for the signal to travel from point $A$ to point $D$ and back to point $A$, In this point vector $v_{1}\left(T_{y+}\right)$ is perpendicular to the $z$ axis.

$$
t_{1}=\frac{L}{c-u_{z}}+\frac{L}{c+u_{z}}=\frac{2 * L^{*} c}{c^{2}-u_{z}^{2}}
$$

The $T_{z_{-}}$represents the moment when earth reaches to the point $Z_{-}$, and let $u_{2}$ represent the orthogonal projection of vector $v_{1}\left(T_{z^{-}}\right)$on the $\boldsymbol{z}$ axis. 


$$
\begin{aligned}
& \angle\left(\mathbf{z}^{+}, \boldsymbol{v}_{1}\left(T_{z-}\right)\right)=\Pi-\varphi_{1} \\
& \begin{aligned}
u_{2} & =\operatorname{proj}_{z}\left(\boldsymbol{v}_{1}\left(T_{z^{-}}\right)\right)=\left|\boldsymbol{v}_{1}\left(T_{z^{-}}\right)\right| * \cos \left(\angle\left(\mathbf{z}^{+}, \boldsymbol{v}_{1}\left(T_{z^{-}}\right)\right)\right) \\
& =\left|\boldsymbol{v}_{1}\left(T_{z^{-}}\right)\right| * \cos \left(\Pi-\varphi_{1}\right) \\
& =-\left|\boldsymbol{v}_{1}\left(T_{z^{-}}\right)\right| * \sin \left(\varphi_{0}\right)
\end{aligned}
\end{aligned}
$$

Let $t_{2}$ represent the time that it takes for the signal to travel from the point $A$ to the point $D$ and back to the point $A$.

$$
t_{2}=\frac{L}{c-\left(u_{z}+u_{2}\right)}+\frac{L}{c+\left(u_{z}+u_{2}\right)}=\frac{2 * L^{*} c}{c^{2}-\left(u_{z}+u_{2}\right)^{2}}
$$

Let $t_{3}$ represent the time that it takes for the signal to travel from point $A$ to point $D$ and back to point $A$, when earth reaches to the point $Y_{-}$. In this point vector $\boldsymbol{v}_{1}\left(T_{y-}\right)$ is perpendicular to the $\mathbf{z}$-axis.

$$
t_{3}=\frac{L}{c-u_{z}}+\frac{L}{c+u_{z}}=\frac{2 * L^{*} c}{c^{2}-u_{z}^{2}}
$$

Replacing

$$
c_{i}=\frac{2 L}{t_{i}}, \quad i\{0,1,2,3\}
$$

From ((2) and (3) ) it follows

$$
\begin{gathered}
c^{2}-\left(u_{z}+u_{0}\right)^{2}=c_{0}{ }^{*} c \\
c^{2}-u_{z}^{2}=c_{1}{ }^{*} c
\end{gathered}
$$

After subtracting (7) from (8) we will obtain

$$
2 * u_{0} * u_{z}+\left(c_{0}-c_{1}\right) * c=-u_{0}^{2}
$$

Analogously from ((5) and (6)) it follows that

$$
2 * u_{2} * u_{z}+\left(c_{2}-c_{3}\right) * c=-u_{2}^{2}
$$

In that way we've got a linear system of two equations with two unknowns $u_{z}$ and $c$

$$
\begin{gathered}
\Delta=2 *\left|\begin{array}{ll}
u_{0} & \left(c_{0}-c_{1}\right) \\
u_{2} & \left(c_{2}-c_{3}\right)
\end{array}\right| \\
\Delta_{c}=-2 *\left|\begin{array}{ll}
u_{0} & u_{0}^{2} \\
u_{2} & u_{2}^{2}
\end{array}\right| \\
\Delta_{z}=-\left|\begin{array}{ll}
u_{0}^{2} & \left(c_{0}-c_{1}\right) \\
u_{2}^{2} & \left(c_{2}-c_{3}\right)
\end{array}\right|
\end{gathered}
$$

Let $c$ represent a mean value of the sequence $\left\{c_{i}\right\}$

$$
\begin{gathered}
\boldsymbol{c}=\frac{c_{0}+c_{1}+c_{2}+c_{3}}{4} \\
V_{c}=\frac{\sum_{i=0}^{3}\left(c_{i}-\boldsymbol{c}\right)^{2}}{4}
\end{gathered}
$$

Let $\sigma_{c}$ denotes standard deviation for $c$, and let $\varepsilon$ is some small positive 
number

$$
\sigma_{c}=\sqrt{V_{c}}
$$

If $\left(\sigma_{c}<\varepsilon\right)$ then it follows

$$
c_{z}=\boldsymbol{C}
$$

$u_{z} \equiv 0$ (in this case value of $u_{z}$ is not determined, thus value 0 was assigned to the $\left.u_{z}\right)$

If $\left(\sigma_{c} \geq \varepsilon\right)$ and $(\Delta \# 0)$ then we can solve this linear system and get

$$
\begin{aligned}
& c_{z}=\frac{\Delta_{c}}{\Delta} \\
& u_{z}=\frac{\Delta_{z}}{\Delta}
\end{aligned}
$$

\section{The Measurements along the $x$ and $y$ Axes}

Measurements along the $\boldsymbol{x}$ and $\boldsymbol{y}$ axes are a little bit more complicated then the measurements along $\mathbf{z}$-axis. First let the Figure 1 is given.

From the Figure 1, we will derive Table 1 as follows.

\begin{tabular}{|c|c|c|c|c|c|c|c|c|c|c|c|c|}
\hline & \multicolumn{2}{|c|}{$X_{+}\left(\equiv Z_{+}\right)$} & \multicolumn{4}{|c|}{$Y_{+}$} & \multicolumn{3}{|c|}{$X_{-}\left(\equiv Z_{-}\right)$} & \multicolumn{3}{|c|}{$Y_{-}$} \\
\hline & Time & S.T. & $\mathrm{R}$ & Time & S.T. & $\mathrm{R}$ & Time & S.T. & $\mathrm{R}$ & Time & S.T. & $\mathrm{R}$ \\
\hline$x-$ axis & $T_{x+}+\Delta T_{x+}$ & $s_{0}$ & + & $T_{y+}+\Delta T_{y+}+6$ & $s_{0}+12$ & - & $T_{x-}+\Delta T_{x-}$ & $s_{0}+12$ & - & $T_{y-}+\Delta T_{y-}+6$ & $S_{0}$ & + \\
\hline$y$-axis & $T_{x+}+\Delta T_{x+}+6$ & $s_{0}+6$ & + & $T_{y+}+\Delta T_{y+}$ & $s_{0}+6$ & + & $T_{x-}+\Delta T_{x-}+6$ & $s_{0}+18$ & - & $T_{y-}+\Delta T_{y-}$ & $s_{0}+18$ & - \\
\hline
\end{tabular}

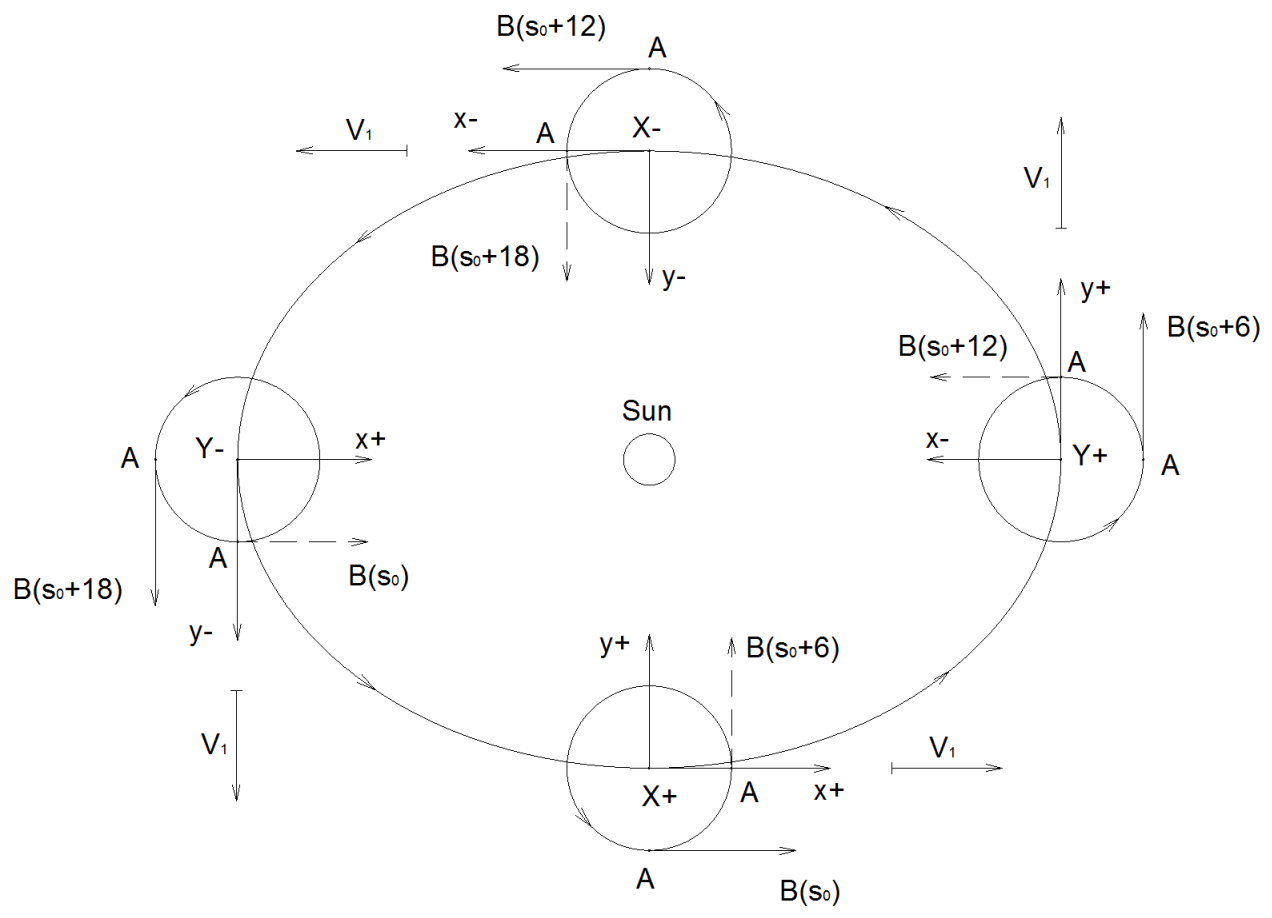

Figure 1. Plane xy at the four different points during the experiments.

Table 1. The scheme for conducting the experiments in the west-east direction. 
Now we will analyze the data given by Table 1 and explain how to use them in our experiment. Suppose that $A B$ is some given line on the earth's equator in the direction west-east. The length of the line $A B$ is equal to $L$.

The earth is rotating about $z$-axis, thus direction of the line $A B$ is constantly changing. During the period of $24 \mathrm{~h} A B$ rotates at $x y$ plane about the origin $O$ by $2 * \Pi$. Thus, for any non-zero vector at the plane $x y$ there is a moment when $A B$ and that vector have the same direction.

At the moment $T_{x+}$ the center of the earth is about to reach to the point $X_{+}$.

We have to take a measurement at the moment when $\mathrm{AB}$ and $x^{+}$have the same direction, thus we have to correct the time $T_{x+}$ by some $\Delta T_{x+}\left(\left|\Delta T_{x+}\right|<12 \mathrm{~h}\right)$.

Time $\left(T_{x+}+\Delta T_{x+}\right)$ is equivalent to the some sidereal time denoted by $s_{0}$, that will serve as a referential time.

$$
\left(T_{x+}+\Delta T_{x+}\right) \sim s_{0}
$$

The column "S.T. " represents sidereal time when a measurement was made.

We are assuming that following equivalence is valid

$$
(s+k) \sim(s+24 \mathrm{~h}-k)
$$

where $s, k$ denote some sidereal day time

In case that the velocity of the earth rotation around its axis has the same direction as positive axis $\left(\boldsymbol{x}^{+}\right.$or $\left.\boldsymbol{y}^{+}\right)$the value of column " $R^{\text {" }}$ is equal to " + " otherwise if it has the same direction as $\boldsymbol{x}^{-}$or $\boldsymbol{y}^{-}$then the value of column " $R$ " will be equal to "-". Correcting the time $T_{x+}$ we also have to correct the angle $\varphi_{0}$ (angle between vector $\boldsymbol{v}_{1}(T)$ and $\boldsymbol{x}^{+}$) by some $\Delta \varphi$.

At this moment $\left(T_{x_{+}}+\Delta T_{x^{+}}\right)$, we are going to send a signal from the point $A$ towards the point $B$. After signal reaches the point $B$, it will be reflected back to the point $A$.

Let $t$ represent the time that it takes for the signal to travel from the point $A$ to the point $B$ and back to the point $A$.

In that way the measurement along $\boldsymbol{x}^{+}$axis at the point $X_{+}$was made.

At the moment $\left(s_{0}\right) \boldsymbol{y}$-axis is perpendicular to $A B$, the $\boldsymbol{y}$-axis which is considered as a vector is fixed. Thus to make measurement along $\boldsymbol{y}$-axis, we have to wait for the next 6 (sidereal) hours in order to line $A B$ and $y^{+}$at the same direction.

Solar time $\left(T_{x+}+\Delta T_{x+}+6 \mathrm{~h}\right)$ is equvivalent to the sidereal time $\left(s_{0}+6 \mathrm{~h}\right)$

At the moment $\left(T_{x+}+\Delta T_{x+}+6 \mathrm{~h}\right)$ we are going to send a signal from the point $A$ towards the point $B$. After signal reaches the point $\mathrm{B}$ it will be reflected back towards the point $A$.

In that way the masurements at the point $X_{+}$along $\boldsymbol{x}^{+}$and $\boldsymbol{y}^{+}$axes have been made.

In a similar way we will make measurements at the point $Y_{+}$along $\boldsymbol{x}^{-}$and $y^{+}$axes, at the point $X_{-}$along $x^{-}$and $y^{-}$axes and at the point $Y_{-}$along $\boldsymbol{x}^{+}$and $\boldsymbol{y}^{-}$axes.

\section{The Results of the Measurements along the X-Axis}

Let earth's equatorial rotational speed at the point $A$ is denoted by $u_{r}$. 
Let $u_{0}$ represent a sum of the orthogonal projection of the vector $v_{1}\left(T_{X+}+\Delta T_{X+}\right)$ on the $\mathrm{x}$ axis and $u_{r}$.

$$
\begin{aligned}
& \left(T_{x+}+\Delta T_{x+}\right) \sim s_{0} \\
& \angle\left(\boldsymbol{x}^{+}, \boldsymbol{v}_{1}\left(T_{x+}+\Delta T_{X+}\right)\right)=\varphi_{0}+\Delta \varphi \\
u_{0}= & \operatorname{proj}_{x}\left(v_{1}\left(T_{x+}+\Delta T_{X+}\right)\right)+u_{r} \\
= & \left|v_{1}\left(T_{x+}+\Delta T_{X+}\right)\right| * \cos \left(\varphi_{0}+\Delta \varphi\right)+u_{r} \\
& \left(\left|v_{1}\left(T_{x+}\right)\right| * \cos \left(\varphi_{0}\right) \approx 27.5118 \mathrm{~km} / \mathrm{sec}\right)
\end{aligned}
$$

Let $t_{0}$ represent the time that it takes for the signal to travel from the point $A$ to the point $B$ and back to the point $A$.

$$
t_{0}=\frac{L}{c-\left(u_{0}+u_{x}\right)}+\frac{L}{c+\left(u_{0}+u_{x}\right)}=\frac{2 * L^{*} c}{c^{2}-\left(u_{0}+u_{x}\right)^{2}}
$$

Now we are going to make a second measuring along the $\boldsymbol{x}$ axis at the point $Y_{+}$, a moment when the earth reaches to the point $Y_{+}$is denoted by $T_{y+}$

First we have to make a measurement along $y^{+}$axis. That will happen at the moment $\left(T_{y+}+\Delta T_{y+}\right) \sim s_{0}+6 \mathrm{~h}$. Six (sidereal) hours later measurements along $\boldsymbol{x}$-axis will be performed.

$$
\begin{aligned}
& \left(T_{y+}+\Delta T_{y+}+6 \mathrm{~h}\right) \sim s_{0}+12 \mathrm{~h} \\
& \angle\left(\boldsymbol{x}^{+}, v_{1}\left(T_{y+}+\Delta T_{y+}+6 \mathrm{~h}\right)\right)=\frac{\pi}{2}+\Delta \varphi
\end{aligned}
$$

Let $u_{1}$ represent a sum of the orthogonal projection of the vector $v_{1}\left(T_{y+}+\Delta T_{y+}+6 \mathrm{~h}\right)$ on the $\mathbf{x}$ axis and $u_{r}$

$$
\begin{aligned}
u_{1} & =\operatorname{proj}_{x}\left(v_{1}\left(T_{y+}+\Delta T_{y+}+6 \mathrm{~h}\right)\right)-u_{r} \\
& =\left|v_{1}\left(T_{y+}+\Delta T_{y+}+6 \mathrm{~h}\right)\right| * \cos \left(\frac{\pi}{2}+\Delta \varphi\right) \\
& =-\left|v_{1}\left(T_{y+}+\Delta T_{y+}+6 \mathrm{~h}\right)\right| * \sin (\Delta \varphi)-u_{r}
\end{aligned}
$$

Let $t_{1}$ represent the time that it takes for the signal to travel from the point $A$ to the point $B$ and back to the point $A$ at the moment $\left(T_{y+}+\Delta T_{y+}+6 \mathrm{~h}\right)$

$$
t_{1}=\frac{L}{c-\left(u_{1}+u_{x}\right)}+\frac{L}{c+\left(u_{1}+u_{x}\right)}=\frac{2 * L^{*} c}{c^{2}-\left(u_{1}+u_{x}\right)^{2}}
$$

The third measurement is going to be taken when the center of the earth is about to reach to the point $X_{-}$. That moment is denoted by $T_{x_{-}}$.

The measurement will be made at the moment when $A B$ have the same direction as $\boldsymbol{x}^{-}$.

Let $u_{2}$ represent a sum of the orthogonal projection of vector $v_{1}\left(T_{x_{-}}+\Delta T_{x_{-}}\right)$ on the $\boldsymbol{x}$ axis and $u_{r}$

$$
\begin{aligned}
& \left(T_{x_{-}}+\Delta T_{x_{-}}\right) \sim\left(s_{0}+12 \mathrm{~h}\right) \\
& \angle\left(\boldsymbol{x}^{+}, v_{1}\left(T_{x_{-}}+\Delta T_{x^{-}}\right)\right)=\Pi-\varphi_{0}+\Delta \varphi
\end{aligned}
$$




$$
\begin{aligned}
u_{2} & =\operatorname{proj}_{x}\left(v_{1}\left(T_{x-}+\Delta T_{x-}\right)\right)-u_{r} \\
& =\left|v_{1}\left(T_{x-}+\Delta T_{x-}\right)\right| * \cos \left(\Pi-\varphi_{0}+\Delta \varphi\right)-u_{r} \\
& =-\left|v_{1}\left(T_{x-}+\Delta T_{x-}\right)\right| * \cos \left(\varphi_{0}-\Delta \varphi\right)-u_{r}
\end{aligned}
$$

Let $t_{2}$ represent the time that it takes for signal to travel from the point $A$ to the point $B$ and back to the point $A$ at the moment $T_{x_{-}}+\Delta T_{x_{-}}$.

$$
t_{2}=\frac{L}{c-\left(u_{2}+u_{x}\right)}+\frac{L}{c+\left(u_{2}+u_{x}\right)}=\frac{2 * L^{*} c}{c^{2}-\left(u_{2}+u_{x}\right)^{2}}
$$

Now we are going to make a fourth measuring along the $x$-axis. At the moment, denoted by $T_{y-}$, the center of the earth is about to reach to the point $Y_{-}$.

$$
\begin{aligned}
& \left(T_{y-}+\Delta T_{y_{-}}+6 \mathrm{~h}\right) \sim\left(s_{0}\right) \\
& \angle\left(\boldsymbol{x}^{+}, v_{1}\left(T_{y-}+\Delta T_{y_{-}}+6 \mathrm{~h}\right)\right)=\frac{3 \pi}{2}+\Delta \varphi
\end{aligned}
$$

Let $u_{3}$ represent sum of the orthogonal projection of the vector $\boldsymbol{v}_{1}\left(T_{y-}+\Delta T_{y-}+6 \mathrm{~h}\right)$ on the $\boldsymbol{x}$ axis and $u_{r}$.

$$
\begin{aligned}
u_{3} & =\operatorname{proj}_{x}\left(v_{1}\left(T_{y-}+\Delta T_{y-}+6 \mathrm{~h}\right)\right) \\
& =\left|v_{1}\left(T_{y-}+\Delta T_{y-}+6 \mathrm{~h}\right)\right| * \cos \left(\frac{3 \pi}{2}+\Delta \varphi\right)+u_{r} \\
& =\left|v_{1}\left(T_{y-}+\Delta T_{y-}+6 \mathrm{~h}\right)\right| * \sin (\Delta \varphi)+u_{r}
\end{aligned}
$$

Let $t_{3}$ represent the time that it takes for the signal to travel from the point $A$ to the point $B$ and back to the point $A$ at the moment $\left(T_{y+}+\Delta T_{y+}+6 \mathrm{~h}\right)$

$$
t_{3}=\frac{L}{c-\left(u_{3}+u_{x}\right)}+\frac{L}{c+\left(u_{3}+u_{x}\right)}=\frac{2 * L *_{c}}{c^{2}-\left(u_{3}+u_{x}\right)^{2}}
$$

$\Delta \varphi$ varies from one measurement to another. From (2) and (4) it follows that

$$
\begin{aligned}
& c^{2}-\left(u_{0}+u_{x}\right)^{2}=c^{2}-u_{x}^{2}-2 * u_{0} * u_{x}-u_{0}^{2}=\frac{2 L^{*} c}{t_{0}} \\
& c^{2}-\left(u_{1}+u_{x}\right)^{2}=c^{2}-u_{x}^{2}-2 * u_{1} * u_{x}-u_{1}^{2}=\frac{2 L^{*} c}{t_{1}}
\end{aligned}
$$

Replacing $\frac{2 L c}{t_{i}}$ by $c_{i}$ and after subtracting (9) from (10) we get

$$
2 *\left(u_{1}-u_{0}\right) * u_{x}+\left(c_{1}-c_{0}\right) * c=\left(u_{0}^{2}-u_{1}^{2}\right)
$$

Analogously from ((6) and (8)) we obtain that

$$
2 *\left(u_{3}-u_{2}\right) * u_{x}+\left(c_{3}-c_{2}\right) * \boldsymbol{c}=\left(u_{2}^{2}-u_{3}^{2}\right)
$$

In that way we've got a linear system of two equations with two unknowns $u_{x}$ and $\boldsymbol{c}$.

$$
\Delta=2 *\left|\begin{array}{ll}
\left(u_{1}-u_{0}\right) & \left(c_{1}-c_{0}\right) \\
\left(u_{3}-u_{2}\right) & \left(c_{3}-c_{2}\right)
\end{array}\right|
$$




$$
\begin{gathered}
\Delta_{c}=2 *\left|\begin{array}{ll}
\left(u_{1}-u_{0}\right) & \left(u_{0}^{2}-u_{1}^{2}\right) \\
\left(u_{3}-u_{2}\right) & \left(u_{2}^{2}-u_{3}^{2}\right)
\end{array}\right| \\
=2 *\left(u_{1}-u_{0}\right) *\left(u_{3}-u_{2}\right) *\left(u_{0}+u_{1}-u_{2}-u_{3}\right) \\
\Delta_{x}=\left|\begin{array}{ll}
\left(u_{0}^{2}-u_{1}^{2}\right) & \left(c_{1}-c_{0}\right) \\
\left(u_{2}^{2}-u_{3}^{2}\right) & \left(c_{3}-c_{2}\right)
\end{array}\right|
\end{gathered}
$$

Let $c$ represent a mean value of the sequence $\left\{c_{i}\right\}$

$$
\begin{gathered}
\boldsymbol{c}=\frac{c_{0}+c_{1}+c_{2}+c_{3}}{4} \\
V_{c}=\frac{\sum_{i=0}^{3}\left(c_{i}-\boldsymbol{c}\right)^{2}}{4}
\end{gathered}
$$

Let $\sigma_{c}$ denote standard deviation for $\mathcal{c}$, and let $\varepsilon$ is some small positive number

$$
\sigma_{c}=\sqrt{V_{c}}
$$

If $\left(\sigma_{c}<\varepsilon\right)$ then it follows

$$
c_{x}=\boldsymbol{c}
$$

$u_{x} \equiv \mathbf{0}$ (in this case value of $u_{x}$ is not determined, thus value $\mathbf{0}$ was assigned to the $\left.u_{x}\right)$

If $\left(\sigma_{c} \geq \varepsilon\right)$ and $(\Delta \# 0)$ then we can solve this linear system and get

$$
\begin{aligned}
& c_{x}=\frac{\Delta_{c}}{\Delta} \\
& u_{x}=\frac{\Delta_{x}}{\Delta}
\end{aligned}
$$

\section{Measurements along the Y-Axis}

Our discussion starts from the point $\boldsymbol{Y}_{+}$and move in an counterclockwise direction (Figure 1) to the next point $X_{-}$.

Let $u_{0}$ represent a sum of the orthogonal projections of vector $v_{1}\left(T_{y+}+\Delta T_{y_{+}}\right)$ on the $y$ axis and $u_{r}$

$$
\begin{aligned}
& \left(T_{y+}+\Delta T_{y+}\right) \sim\left(s_{0}+6 \mathrm{~h}\right) \\
& \angle\left(y^{+}, v_{1}\left(T_{y+}+\Delta T_{y+}\right)\right)=0+\Delta \varphi \\
u_{0}= & \operatorname{proj}_{y}\left(v_{1}\left(T_{y+}+\Delta T_{y+}\right)\right)+u_{r} \\
= & \left|v_{1}\left(T_{y+}+\Delta T_{y+}\right)\right| * \cos (0+\Delta \varphi)+u_{r} \\
= & \left(\left|v_{1}\left(T_{y+}\right)\right| * \cos (0) \approx 30 \mathrm{~km} / \mathrm{sec}\right)
\end{aligned}
$$

Let $t_{0}$ represent the time that it takes for the signal to travel from the point $A$ to the point $B$ and back to the point $A$.

$$
t_{0}=\frac{L}{c-\left(u_{0}+u_{y}\right)}+\frac{L}{c+\left(u_{0}+u_{y}\right)}=\frac{2 * L^{*} c}{c^{2}-\left(u_{0}+u_{y}\right)^{2}}
$$

The second measurement is going to be calculated for the point $X_{-}$ 
Let $u_{1}$ represent a sum of the orthogonal projection of vector $v_{1}\left(T_{x-}+\Delta T_{x-}+6 \mathrm{~h}\right)$ on the $y$ axis and $u_{r}$

$$
\begin{aligned}
& \left(T_{x-}+\Delta T_{x-}+6 \mathrm{~h}\right) \sim\left(s_{0}+18 \mathrm{~h}\right) \\
& \angle\left(y^{+}, v_{1}\left(T_{x-}+\Delta T_{X-}+6 \mathrm{~h}\right)\right)=\frac{\pi}{2}+\Delta \varphi \\
u_{1}= & \operatorname{proj}_{y}\left(v_{1}\left(T_{x-}+\Delta T_{x-}+6 \mathrm{~h}\right)\right) \\
= & \left|v_{1}\left(T_{x-}+\Delta T_{x-}+6 \mathrm{~h}\right)\right| * \cos \left(\frac{\pi}{2}+\Delta \varphi\right)-u_{r} \\
= & -\left|v_{1}\left(T_{x-}+\Delta T_{x-}+6\right)\right| * \sin (\Delta \varphi)-u_{r}
\end{aligned}
$$

Let $t_{1}$ represent the time that it takes for the signal to travel from the point $A$ to the point $B$ and back to the point $A$ at the moment $T_{x_{-}}+\Delta T_{x_{-}}+6 \mathrm{~h}$

$$
t_{1}=\frac{L}{c-\left(u_{1}+u_{y}\right)}+\frac{L}{c+\left(u_{1}+u_{y}\right)}=\frac{2 * L^{*} c}{c^{2}-\left(u_{1}+u_{y}\right)^{2}}
$$

Third measurement is going to be calculated for the point $Y_{-}$.

Let $u_{2}$ represent sum of orthogonal projection of vector $v_{1}\left(T_{y-}+\Delta T_{y_{-}}\right)$on the $y$ axisand $u_{r}$.

$$
\begin{aligned}
& \left(T_{y-}+\Delta T_{y-}\right) \sim\left(s_{0}+18 \mathrm{~h}\right) \\
& \angle\left(\boldsymbol{y}^{+}, \boldsymbol{v}_{1}\left(T_{y-}+\Delta T_{y-}\right)\right)=\Pi-0+\Delta \varphi \\
u_{2}= & \operatorname{proj}_{y}\left(\boldsymbol{v}_{1}\left(T_{y-}+\Delta T_{y-}\right)\right)-u_{r} \\
= & \left|\boldsymbol{v}_{1}\left(T_{y-}+\Delta T_{y-}\right)\right| * \cos (\Pi-0+\Delta \varphi)-u_{r} \\
= & -\left|\boldsymbol{v}_{1}\left(T_{y_{-}}+\Delta T_{y^{-}}\right)\right| * \cos (\Delta \varphi)-u_{r}
\end{aligned}
$$

Let $t_{2}$ represent the time that it takes for the signal to travel from the point $A$ to the point $B$ and back to the point $A$ at the moment $T_{y-}+\Delta T_{y-}$.

$$
t_{2}=\frac{L}{c-\left(u_{2}+u_{y}\right)}+\frac{L}{c+\left(u_{2}+u_{y}\right)}=\frac{2 * L^{*} c}{c^{2}-\left(u_{2}+u_{y}\right)^{2}}
$$

The fourth measurement is going to be calculated for the point $X_{+}$,

$$
\begin{aligned}
& \left(T_{x+}+\Delta T_{x+}+6 h\right) \sim\left(s_{0}+6 \mathrm{~h}\right) \\
& \angle\left(\boldsymbol{y}^{+}, v_{1}\left(T_{x+}+\Delta T_{x+}+6 \mathrm{~h}\right)\right)=\frac{3 \pi}{2}+\Delta \varphi
\end{aligned}
$$

Let $u_{3}$ represent a sum of the orthogonal projection of vector $\boldsymbol{v}_{1}\left(T_{x+}+\Delta T_{x+}+6 \mathrm{~h}\right)$ on the $\boldsymbol{y}$ axis. and $u_{r}$

$$
\begin{aligned}
u_{3} & =\operatorname{proj}_{y}\left(v_{1}\left(T_{x+}+\Delta T_{x+}\right)\right) \\
& =\left|v_{1}\left(T_{x+}+\Delta T_{x+}\right)\right|^{*} \cos \left(\frac{3 \pi}{2}+\Delta \varphi\right)+u_{r} \\
& =\left|v_{1}\left(T_{x+}+\Delta T_{x+}+6\right)\right| * \sin (\Delta \varphi)+u_{r}
\end{aligned}
$$

Let $t_{3}$ represent the time that it takes for the signal to travel from the point $A$ to the point $B$ and back to the point $A$ at the moment $\left(T_{x+}+\Delta T_{x+}+6 \mathrm{~h}\right)$ 


$$
t_{3}=\frac{L}{c-\left(u_{3}+u_{y}\right)}+\frac{L}{c+\left(u_{3}+u_{y}\right)}=\frac{2 * L^{*} c}{c^{2}-\left(u_{3}+u_{y}\right)^{2}}
$$

$\Delta \varphi$ varies from one measurement to another From (2) and (4) it follows that

$$
\begin{aligned}
& c^{2}-\left(u_{0}+u_{y}\right)^{2}=c^{2}-u_{y}^{2}-2 * u_{0} * u_{y}-u_{0}^{2}=\frac{2 L^{*} c}{t_{0}} \\
& c^{2}-\left(u_{1}+u_{y}\right)^{2}=c^{2}-u_{y}^{2}-2 * u_{1} * u_{y}-u_{1}^{2}=\frac{2 L^{*} c}{t_{1}}
\end{aligned}
$$

After subtracting (9) from (10), and replacing $\frac{2 L c}{t_{i}}$ by $c_{i}$

$$
2 *\left(u_{1}-u_{0}\right) * u_{y}+\left(c_{1}-c_{0}\right) * c=\left(u_{0}^{2}-u_{1}^{2}\right)
$$

Analogously from ((6) and (8) ) we will obtain that

$$
2 *\left(u_{3}-u_{2}\right) * u_{y}+\left(c_{3}-c_{2}\right) * c=\left(u_{2}^{2}-u_{3}^{2}\right)
$$

In that way we've got a linear system of two equations with two unknowns $u_{y}$ and $c$.

$$
\begin{gathered}
\Delta=2 *\left|\begin{array}{ll}
\left(u_{1}-u_{0}\right) & \left(c_{1}-c_{0}\right) \\
\left(u_{3}-u_{2}\right) & \left(c_{3}-c_{2}\right)
\end{array}\right| \\
\Delta_{c}=2 *\left|\begin{array}{ll}
\left(u_{1}-u_{0}\right) & \left(u_{0}^{2}-u_{1}^{2}\right) \\
\left(u_{3}-u_{2}\right) & \left(u_{2}^{2}-u_{3}^{2}\right)
\end{array}\right|=2 *\left(u_{1}-u_{0}\right) *\left(u_{3}-u_{2}\right) *\left(u_{0}+u_{1}-u_{2}-u_{3}\right) \\
\Delta_{y}=\left|\begin{array}{ll}
\left(u_{0}^{2}-u_{1}^{2}\right) & \left(c_{1}-c_{0}\right) \\
\left(u_{2}^{2}-u_{3}^{2}\right) & \left(c_{3}-c_{2}\right)
\end{array}\right|
\end{gathered}
$$

Let $c$ represent a mean value of the sequence $\left\{c_{i}\right\}$

$$
\begin{gathered}
\boldsymbol{c}=\frac{c_{0}+c_{1}+c_{2}+c_{3}}{4} \\
V_{c}=\frac{\sum_{i=0}^{3}\left(c_{i}-\boldsymbol{c}\right)^{2}}{4}
\end{gathered}
$$

Let $\sigma_{c}$ denotes standard deviation for $c$, and let $\varepsilon$ is some small positive number

$$
\sigma_{c}=\sqrt{V_{c}}
$$

If $\left(\sigma_{c}<\varepsilon\right)$ then it follows

$$
c_{y}=c
$$

$u_{y} \equiv \mathbf{0} \quad$ (in this case value of $u_{y}$ is not determined, thus value $\mathbf{0}$ was assigned to the $\left.u_{y}\right)$

If $\left(\sigma_{c} \geq \varepsilon\right)$ and $(\Delta \# 0)$ then we can solve this linear system and get

$$
\begin{gathered}
c_{y}=\frac{\Delta_{c}}{\Delta} \\
u_{y}=\frac{\Delta_{y}}{\Delta}
\end{gathered}
$$




\section{Results}

In this section we are going to derive the constant $\mathrm{c}$ and $\boldsymbol{u}$ that are already defined in the Introduction.

The speed of the light, constant $\boldsymbol{c}$ (1.11), has been measured three times.

Let $\boldsymbol{c}$ denotes a mean value and $\boldsymbol{V}_{c}$ denotes its variation.

$$
\begin{gathered}
c=\frac{c_{x}+c_{y}+c_{z}}{3} \\
V_{c}=\frac{\left(c_{x}-c\right)^{2}+\left(c_{y}-c\right)^{2}+\left(c_{z}-c\right)^{2}}{3}
\end{gathered}
$$

Let $\sigma_{c}$ denotes standard deviation for $\mathcal{c}$, and let $\varepsilon$ is some small positive number

$$
\sigma_{c}=\sqrt{V_{c}}
$$

Comparing $\varepsilon$ and $\sigma_{c}$ we will consider four possibilities

i) $\sigma_{c}>\varepsilon$

In this case we will say that our experiment failed, due to some random errors.

ii) $\left(\sigma_{c}<\varepsilon\right)$ and $\left(x=\mathbf{0}\right.$, for $\forall x$ where $\left.x \in\left\{u_{x}, u_{y}, u_{z}\right\}\right)$

We will say that result of the experiment is negative.

The speed of the light $c$ is equal to $c$.

This is the expected result and there are the numerous experiments that indirectly support it, the most known is Michelson-Morley experiment.

iii) $\left(\sigma_{c}<\varepsilon\right)$ and $\left(\exists x \exists y, \ni(x=0\right.$ and $y \# \mathbf{0})$ where $\left.x, y \in\left\{u_{x}, u_{y}, u_{z}\right\}\right)$

In this case we will say that our experiment failed. The results of the experiment lead to the contradiction, because outcome of the experiment can not be 'positive' and 'negative'

iv) $\left(\sigma_{c}<\varepsilon\right)$ and $\left(x \# \mathbf{0}\right.$, for $\forall x$ where $\left.x \in\left\{u_{x}, u_{y}, u_{z}\right\}\right)$

We will say that result of the experiment is positive.

Let $u$ denotes magnitude of the vector $\boldsymbol{u}$

$$
\boldsymbol{u}=\sqrt{u_{x}^{2}+u_{y}^{2}+u_{z}^{2}}
$$

The speed of the light $c$ is equal to $c$, but in this case we are making a certain systematic error in computing the values of $\boldsymbol{u}$ and $\boldsymbol{c}$, because during the period of one year $\boldsymbol{u}$ changes its value.

There are a few of experiments for an example [4] and [5] that support such possibility.

\section{Conclusions}

In this section we are going to discuss under what conditions, using the results from our experiments, we were able to derive the speed of the light in any direction.

Suppose that the two distinct points $A$ and $C$ are given. Is it possible on the basis of the results from our experiments to find out the speed of light in the direction $A C$. The answer is negative. 
So far, in our experiments (we can call them the first set of experiments), we have assumed that lines $A B$ and $A D$ are orthogonal to the earth's gravitational field; however the line $A C$ could lay in any direction.

In order to give more precise answer to the posed question we have to repeat all our experiments assuming that $A B$ and $A D$ are parallel to the earth's gravitational field, in other words, the points $O$ (the center of the earth), $A$ and $B$ are collinear as well the points $O, A$ and $D$. (in this case point $A$ lies on the North or South pole)

Direction of earth's rotation around its axis in the point $A$ is perpendicular to $A B$, therefore in this case it does not affect measuring.

Suppose that the second set of experiments were performed, when $A B$ and $A D$ are parallel to the earth's gravitational field.

Let $c^{\prime}, u_{x}^{\prime}, u_{y}^{\prime}, u_{z}^{\prime}$ represent the results of the second set of the experiments,

Let $c^{\prime}$ correspond to $c, u_{x}^{\prime}$ to $u_{x}, u_{y}^{\prime}$ to $u_{y}$ and $u_{z}^{\prime}$ to $u_{z}$, where $c, u_{x}$, $u_{y}, u_{z}$ represent results from the first set of experiments.

Note that all data were obtained as a result of some measuring, thus when we say $x$ is equal to $y$, it meant that $x$ is equal to $y$ with some permitted error.

Comparing the results from the two sets of experiments, we will analyze three cases

i) $\left(c^{\prime}=c\right)$ and $\left(u_{x}^{\prime}=u_{x}\right)$ and $\left(u_{y}^{\prime}=u_{y}\right)$ and $\left(u_{z}^{\prime}=u_{z}\right)$

In this case we can conclude that speed of light (in the vicinity of the earth) is invariant to the earth's gravitational field.

Absolute velocity denoted by $\boldsymbol{v}_{A}(t)$ that the point $A$ is moving through the space given by expression

$$
\boldsymbol{v}_{A}(t)=\boldsymbol{r}_{A}(t)+\boldsymbol{v}_{1}(t)+\boldsymbol{u}
$$

where

$\boldsymbol{r}_{A}(t)$ - Represent velocity due to earth rotation about its axis.

$\boldsymbol{v}_{1}(t)$ and $\boldsymbol{u}$ are already defined in the Introduction

$$
\text { (365.24219 days } \geq t \geq 0 \text { ) }
$$

Line $A B$ could be considered as a vector where $A$ is its tail and $B$ is its head. In this case cosine between vectors $A B$ and $v_{A}$ is given by the expression.

$$
\cos \left(\overrightarrow{A B}, v_{A}\right)=\frac{\overrightarrow{A B} * v_{A}}{|\overrightarrow{A B}| *\left|v_{A}\right|}
$$

From (1) and (1.10) it follows that

$$
\begin{aligned}
& \boldsymbol{c}_{A B}(t)=\boldsymbol{c}-\left|\boldsymbol{v}_{A}\right| * \cos \left(\overrightarrow{A B}, \boldsymbol{v}_{A}\right)=\mathbf{c}-\boldsymbol{v}_{A} * \frac{\overrightarrow{A B}}{|\overrightarrow{A B}|}=\boldsymbol{c}-\boldsymbol{v}_{A} * \boldsymbol{a}_{0} \\
& \boldsymbol{c}_{A B}(t)=\boldsymbol{c}-\left(\boldsymbol{r}_{A}(t) * \boldsymbol{a}_{0}(\mathrm{t})+\boldsymbol{v}_{1}(t) * \boldsymbol{a}_{0}(\mathrm{t})+\boldsymbol{u}^{*} \boldsymbol{a}_{0}(t)\right)
\end{aligned}
$$

where $\overrightarrow{A B}=|\overrightarrow{A B}| * \boldsymbol{a}_{0}(t)$

ii) $\left(c^{\prime} \# c\right)$ and $\left(u_{x}^{\prime}=u_{x}\right)$ and $\left(u_{y}^{\prime}=u_{y}\right)$ and $\left(u_{z}^{\prime}=u_{z}\right)$

In this case we can conclude that, besides the earth movement through the space, earth gravitational field affects measure the speed of light as well. 
This result is beyond the scope of this paper.

iii) $\left(u_{x}^{\prime} \# u_{x}\right)$ or $\left(u_{y}^{\prime} \# u_{y}\right)$ or $\left(u_{z}^{\prime} \# u_{z}\right)$

In this case we will say that results of our experiments are inconsistent and declare that our experiment failed.

In the paper [1] we made clear difference between the speed of the light $c$ (defined by 1.11), the speed of the signal denoted by $c_{A B}$ from the point $A$ to the point $B$, and the speed of the signal denoted by $c_{B A}$ from the point $\mathrm{B}$ to the point $A$

Definition of speed of light $c$ given by (1.11) is considered obsolete. The constant $\mathbf{c}$ is presently defined in the following way.

"The speed of light in vacuum, commonly denoted $\mathbf{c}$, is a universal physical constant important in many areas of physics. Its exact value is $299,792,458$ metres per second..." [6].

And definition for the unit of length "metre" is in [3].

"The metre is the length of the path travelled by light in the vacuum during a time in terval of $1 / 299,792,459$ second."

This definition for $\boldsymbol{c}$ assumes that values $\boldsymbol{c}, \boldsymbol{c}_{A B}, \boldsymbol{c}_{A B}$ are equal to each other; otherwise one 'metre' in the direction $A B$ would be different from one 'metre' in direction $B A$.

However, none of the experiments which had a goal to measure the speed of the light did directly prove that.

The question is if $\boldsymbol{c}_{A B}, \boldsymbol{c}_{B A}$, are not equal to each other about how is it possible to measure $c$ with such high accuracy. In order to give a precise answer we should know the relation between the distances that signal had travelled during the experiment and clock accuracy (The principle given by 2.5 ).

At the end we will make a final conclusion.

In the case that outcome of the experiments described in the paper [1], was positive we would be able to

-Prove anisotropy of the speed of the light.

- Measure the speed of light (with a certain error).

-Find out the speed (scalar) that Solar System moves through the space.

In the case that outcome of the experiments from this paper was positive we would be able to

-Find out the velocity that Solar System moves through the space.

-Find out the velocity that Milky Way galaxy moves through the space, assuming that the velocity Solar System moving about the centre of Milky Way galaxy is known .

-Determine how gravitational force affects the speed of light.

-Redefine the definitions for the metre [3] and the constant c [6].

-Measure the speed of the light $c$ defined by (1.11) (with a certain error).

\section{Acknowledgements}

Author would like to thank to Ovidius Botezatu for making the drawings for this paper. 


\section{References}

[1] Čojanović, M. (2016) The Impact of the earth's Movement through the Space on Measuring the Velocity of Light. Journal of Applied Mathematics and Physics, 4, 1168-1178. https://doi.org/10.4236/jamp.2016.46121

[2] Wikipedia (2015) Earth-centered inertia. https://en.wikipedia.org/wiki/Earth-centered inertial

[3] Bureau International des poids et mesures (2014) Base units. http://www.bipm.org/en/measurement-units/base-units.html

[4] Reginald, T.C. (2006) The Roland De Witte 1991 Detection of Absolute Motion and Gravitational Waves.

[5] Stefan, M. (1979) Measurement of the Labaratory's absolute velocity.

[6] Wikipedia (2004) Speed of light. https://en.wikipedia.org/wiki/Speed of light

\section{Submit or recommend next manuscript to SCIRP and we will provide best} service for you:

Accepting pre-submission inquiries through Email, Facebook, LinkedIn, Twitter, etc. A wide selection of journals (inclusive of 9 subjects, more than 200 journals)

Providing 24-hour high-quality service

User-friendly online submission system

Fair and swift peer-review system

Efficient typesetting and proofreading procedure

Display of the result of downloads and visits, as well as the number of cited articles Maximum dissemination of your research work

Submit your manuscript at: http://papersubmission.scirp.org/

Or contact jamp@scirp.org 